\title{
Effects of Alkaline Earth Metal Amides on Ru in Catalytic Ammonia Decomposition
}

Pei Yu ${ }^{a b}$, Jianping Guo ${ }^{* a}$, Lin Liu $^{a}$, Peikun Wang ${ }^{a b}$, Fei Chang ${ }^{a b}$, Han Wang ${ }^{a b}$, Xiaohua Ju ${ }^{a}$ and Ping Chen ${ }^{*}$ acd

a Dalian Institute of Chemical Physics, Chinese Academy of Sciences, Dalian 116023, People's Republic of China

b University of Chinese Academy of Sciences, Beijing 100049, People's Republic of China

c State Key Laboratory of Catalysis, Dalian Institute of Chemical Physics, Chinese Academy of Sciences, Dalian 116023, People's Republic of China

d Collaborative Innovation Center of Chemistry for Energy Materials, Dalian Institute of Chemical Physics, Chinese Academy of sciences, Dalian 116023, People's Republic of China 


\section{Ru dispersion calculation method}

These composite catalysts can not be carried out chemisorption measurements due to the particularity of materials. So we used TEM to estimate the dispersion of Ru nanoparticle and TOF value of catalyst. The Ru dispersion calculation method is given as follows:

According to TEM results, our supported Ru belongs to hexagonal crystal system, with primitive cell parameters $\mathrm{a}=\mathrm{b}=0.271 \mathrm{~nm}, \mathrm{c}=0.428 \mathrm{~nm}$

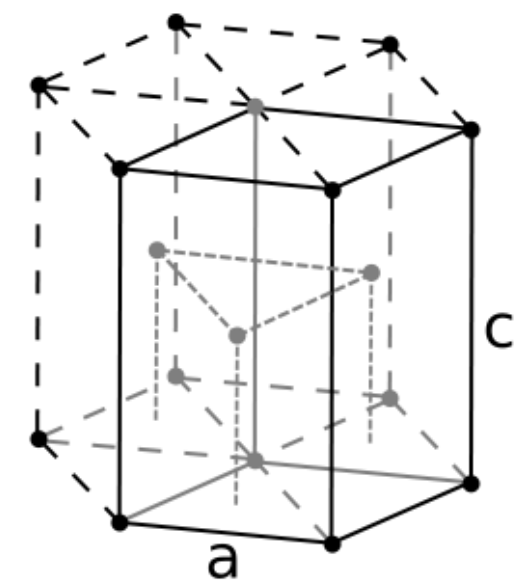

Hexagonal close packed crystal structure

A unit cell of $\mathrm{Ru}$ contains $\mathrm{n}_{1}=3+1 / 2 * 2+1 / 6 * 12=6 \mathrm{Ru}$ atoms with unit cell volume of $\mathrm{V}_{0}$

$=1.5 \sqrt{ } 3 \mathrm{a}^{2} \mathrm{c}=0.082 \mathrm{~nm}^{3}$. So one Ru atom takes up $\mathrm{V}_{1}=\mathrm{V}_{0} / \mathrm{n}_{1}=0.014 \mathrm{~nm}^{3}$ volume.

As a rough estimation, all $\mathrm{Ru}$ particles are assumed to be cubic shape with a size of $\mathrm{d}=2 \mathrm{~nm}$ (mean particle size from TEM result). For simplicity, different exposed crystal planes of Ru have the same densities of surface Ru atoms as (001) crystal plane.

Then the total volume of one Ru cube is $V_{2}=d^{3}=8 \mathrm{~nm}^{3}$,

Therefore, one $\mathrm{Ru}$ cube contains total $\mathrm{N}=\mathrm{V}_{2} / \mathrm{V}_{1}=571 \mathrm{Ru}$ atoms.

Surface area of (001) plane in a unit cell is $\mathrm{S}_{0}=(1.5 \sqrt{ } 3) \mathrm{a}^{2}=0.19 \mathrm{~nm}^{2}$ and (001) plane contains $\mathrm{n}_{2}=$ $1 / 3 * 6+1=3 \mathrm{Ru}$ atoms, so one surface Ru atom takes up $S_{1}=S_{0} / n_{2}=0.064 \mathrm{~nm}^{2}$.

The total surface area of one Ru cube is $S_{2}=6 d^{2}=24 \mathrm{~nm}^{2}$.

Thus, one Ru cube contains total $n=S_{2} / S_{1}=375 \mathrm{Ru}$ atoms.

Therefore, $\mathrm{Ru}$ dispersion is calculated to be: $\mathrm{n} / \mathrm{N}=66 \%$

TOF value can be easily calculated according to Ru dispersion value. 


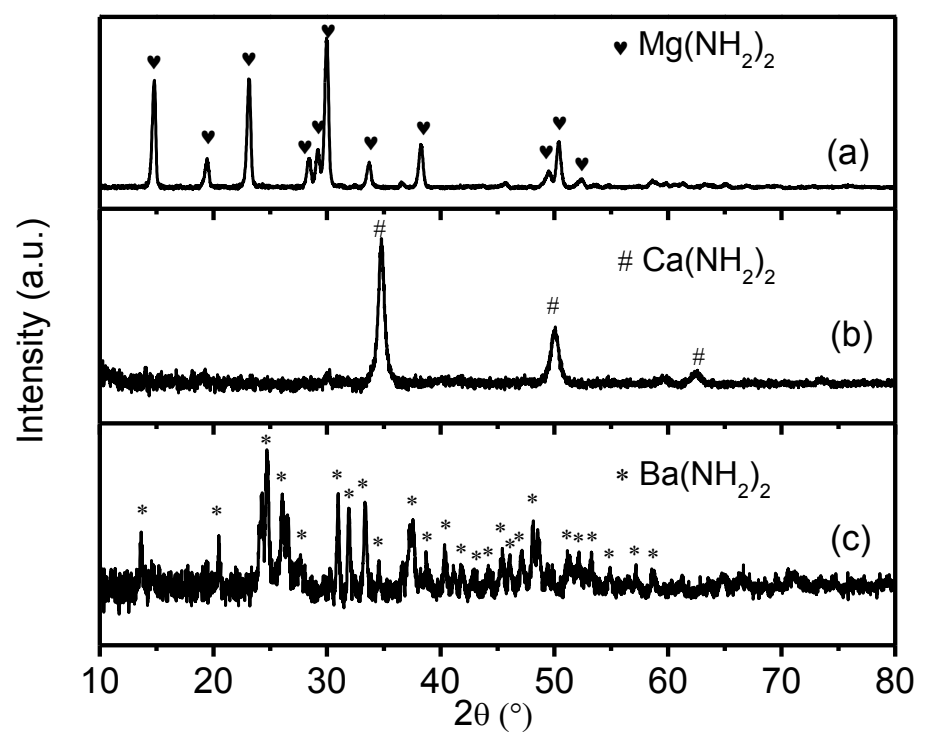

Figure S1. XRD patterns of self-made (a) $\mathrm{Mg}\left(\mathrm{NH}_{2}\right)_{2}$, (b) $\mathrm{Ca}\left(\mathrm{NH}_{2}\right)_{2}$, and (c) $\mathrm{Ba}\left(\mathrm{NH}_{2}\right)_{2}$ samples.

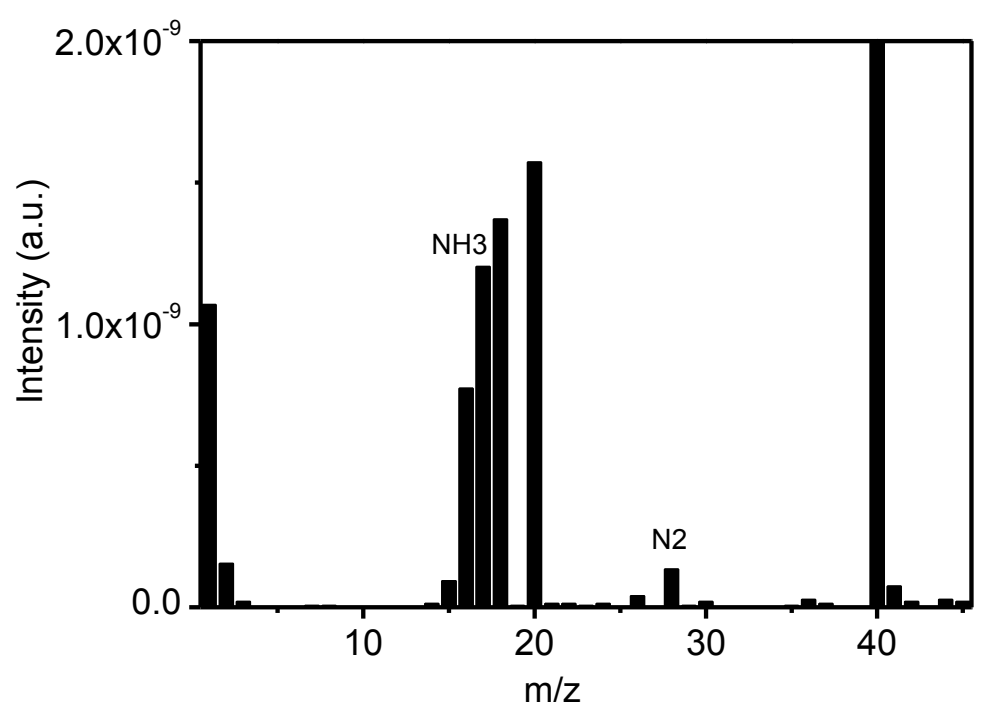

Figure S2. MS analyses of collected gaseous products after ball-milling $\mathrm{RuCl}_{3}$ with $\mathrm{Ca}\left(\mathrm{NH}_{2}\right)_{2}$. 


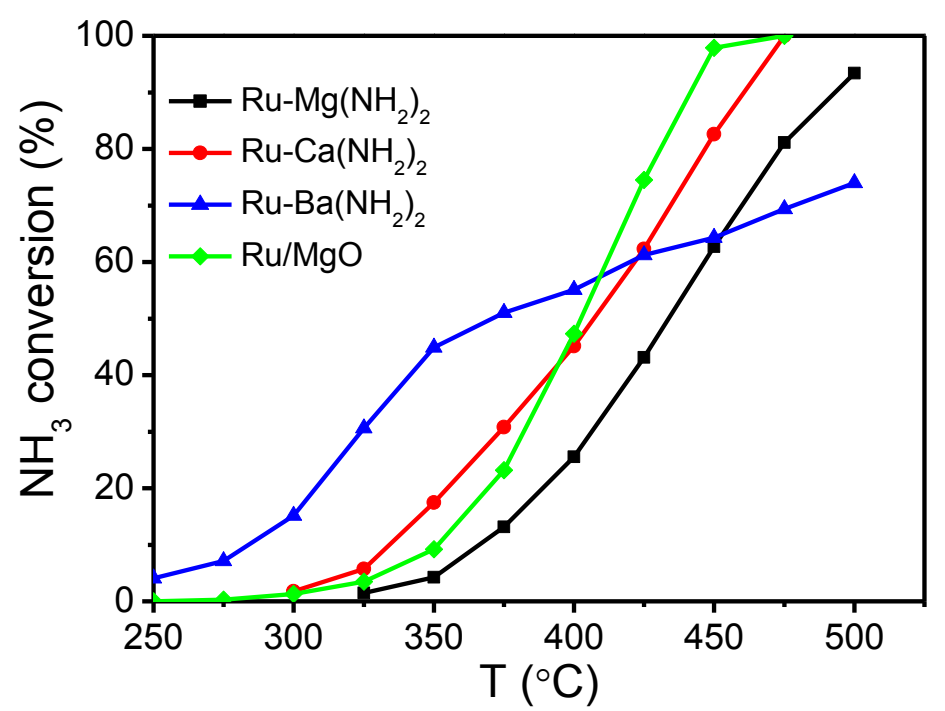

Figure S3. Temperature dependence of $\mathrm{NH}_{3}$ conversion over Ru-based catalysts. Reaction conditions: sample loading - $30 \mathrm{mg}, \mathrm{C}_{\mathrm{NH} 3}-5 \mathrm{vol} \% \mathrm{NH}_{3} / \mathrm{Ar}$, flow rate $-30 \mathrm{~mL} / \mathrm{min}$.

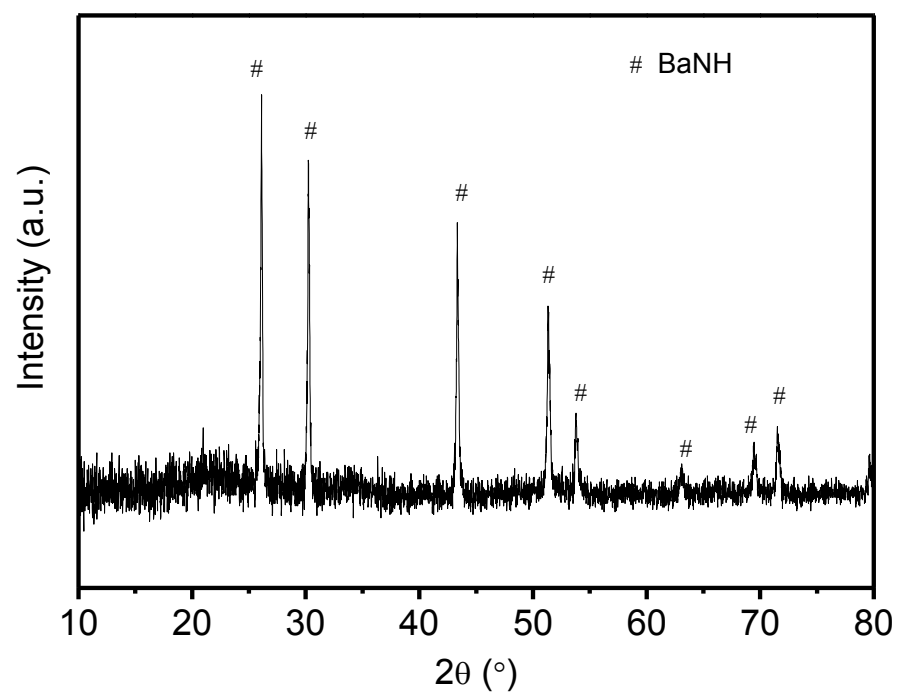

Figure $\mathrm{S} 4$. XRD pattern of $\mathrm{Ba}\left(\mathrm{NH}_{2}\right)_{2}$ sample collected after TPR to $500{ }^{\circ} \mathrm{C}$. 


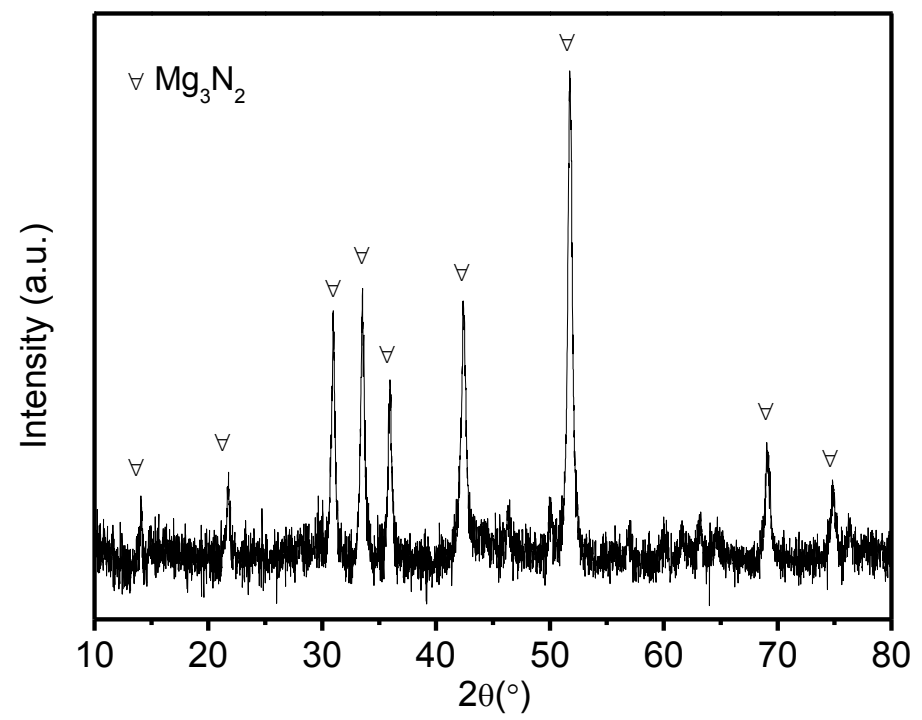

Figure S5. XRD pattern of decomposition product of $\mathrm{Mg}\left(\mathrm{NH}_{2}\right)_{2}$ at $500{ }^{\circ} \mathrm{C}$. 